\title{
Tracking of Three Variants of Transition-Free Lattices for a Proton Driver
}

\author{
Bela Erdelyi* and Carol Johnstone*
}

${ }^{*}$ Fermi National Accelerator Laboratory, P.O. Box 500, MS 220, Batavia, IL, 60510

\begin{abstract}
Transition-free lattices are favored as possible realization of proton drivers. Several variants have been proposed, some of which have considerably different behavior. One of the main quantities used to characterize this behavior is the short term dynamic aperture (DA). In this note we study three different variants of such lattices, and show that the differences in DA among the lattices essentially disappear as soon as magnet multipole errors are included in the simulation. The tracking results can be understood in terms of the normal form based amplitude dependent tune shift and resonance strength coefficients.
\end{abstract}

\section{TRANSITION-FREE LATTICES}

Transition is defined as the point during acceleration where there is no deviation in the revolution period as a function of momentum. Transitionless machines avoid transition either by injecting beam above the transition energy; that is, the pathlength of the higher momentum particles is sufficiently long that they always arrive later than the synchronous particle (relative to the rf waveform), or, more commonly, by shortening the pathlength of high-momentum particles via lattice design until a shorter revolution period is maintained throughout the acceleration cycle. In general, control over the transition energy is achieved by changing the dispersion function and the location of dipoles with respect to the dispersion function. This is easy to see, since the higher the dispersion, the larger the orbit is offset radially from the onmomentum orbit and, consequently, the longer the traversal time for higher momentum particles (conversely, it becomes shorter for low-momentum ones).

In general, transition is higher in energy the shorter the arc cell length when the phase advance per cell is kept constant. For the PD energies studied here, the cell length would require an inter-quadrupole spacing of only about $4 \mathrm{~m}$ which is clearly not an option after considering injection and extraction. The other approaches to controlling transition are "missing-dipole" and "strong focussing" techniques. The missing dipole is based on the standard FODO arc module, but dipoles are removed from high dispersion locations. The PD arc modules studied here are formed from a trio of FODO cells with dipoles removed from the center FODO cell. The effect is to create a dispersion function across the three cell unit which, when integrated with $1 / \rho$ across the length of the remaining dipoles, reduces the slip factor, $\eta$ when compared with a normal three cell unit. If enough empty space is available, the dispersion function can be driven negative for some fraction of the module length. A second way to control the dispersion function is by increasing the quadrupole focussing strength. One example of increased quadupole strengths has already been mentioned-the shortened standard cell. A second way to increase focussing strength is to create a horizontal lower-beta region at the center of a focussing quadrupole by inserting a quadrupole triplet or doublet and a short drift. Outside of the low-beta region, the beta functions are matched to normal FODO cell ones, making the insertion transparent in the linear optics (especially if the phase advance across the insertion is close to $\pi$ ). The peak beta functions are the same as the standard FODO cell, and the fractional cell phase advances can be made similar; hence the optical properties are also not strongly different. In this approach long regions of negative dispersion can be created, and, by propitiously locating dipoles, the transition can often be driven imaginary (transition does not occur at any energy indicating pathlength decreases with momentum).

The missing dipole is the simplest structure of the two, but generally has lower dipole packing so that the arcs must be longer, and realizes a more limited range in $\gamma_{t}$. Dispersion suppression for straights is standard or induced with a dispersion wave by instituting an integer phase advance across the arcs. The strong-focussing lattice has the more complicated low-beta insertion, but exhibits standard FODO cell dipole packing and arcs which are more compact. Also, dispersion suppression is usually more efficient in terms of length requirements. For similar peak beta functions, the strong-focussing lattices have higher transitions (unless significant bend is removed in the missing dipole case). PD lattices have been designed with both types of transition control, but the studies which follow concentrate on the "missing dipole" types which have so far been proposed for the new generation of PDs, mainly because of their close resemblance to conventional synchrotron lattices.

However, in the following, an abnormal sensitivity to 
the positions of the chromatic-correction sextupoles was discovered in the transition-free lattices proposed which resulted in a local nonlinear cancellation of geometric aberrations and seeming enhancement of dynamic performance. This artifact associated with the specific layout of the arc module was verified by constructing the same lattice using conventional FODO cells (which were not transition-free), and placing the chromatic-correction sextupoles as in the transition-free case. Hence the effect could be pinpointed to the particular placement of the chromatic-correction sextupoles. (For example, standard FODO cells with sextupole correctors every half cell do not show the same sensitivities and depowering of a sextupole family always results in an increase in dynamic aperture for an ideal, or error-free, lattice.) When standard field errors are introduced, the advertised properties (particularly the large dynamic apertures) of these sensitive lattices were found to deteriorate substantially, making the different versions almost identical in performance. The details leading to these conclusions are presented below.

\section{RESULTS}

As mentioned in the previous section, we tracked three versions of transition-free lattices for proton drivers. The lattices are termed hereafter: FODO, R. FODO, and PD which represent an arc module composed of 3 standard FODO cells, an identical three-cell FODO module, but with rearranged sextupoles to match the PD locations, and the PD arc module, which has missing dipoles, respectively. (The standard FODO module has chromatic correction sextupoles located near each quadrupole, while the rearranged FODO and PD modules have sextupoles in the center FODO cell only). The ideal lattices were tracked with the full complement of chromatic correction sextupoles, then with horizontal sextupoles turned off, and, finally, with a realistic set of multipole errors.

The code COSY Infinity was used to track [1, 2, 3]. We note that COSY is a single particle map code, in which the correct differential equations are used (including the kinematic effects and thick elements) with a high precision integration algorithm. The fringe fields were turned off (sharp cutoff approximation). The tracking was performed with an order 18 one-turn transfer map, by first employing a quick search for the approximate DA using the truncated Taylor part, followed by fine tuning with the optimally symplectified EXPO map $[4,5]$. It is worth mentioning that, while order 7 effects were still strong in the case of the ideal lattices, order 9 and higher effects were almost always negligible. With errors turned on, higher order effects became important.

The initial conditions for tracking were set up on a grid in polar coordinates in the $x-y$ plane with steps of $0.5 \mathrm{~cm}$ along seven directions with polar angles: $0^{\circ}, 15^{\circ}, 30^{\circ}, 45^{\circ}, 60^{\circ}, 75^{\circ}$, and $90^{\circ}$. The transverse momenta were set to zero and tracking preformed for 1000 turns. As DA along each angle the largest value of radial distance was taken, for which all particles with smaller radii survived.

\section{DA of the ideal lattices}

First, we tracked the ideal lattices. In the following, the results are presented along three angles only, because the DAs in other directions follow from an interpolation of the values shown in the tables below.

As shown in Table 1, the ideal lattices show widely different behavior: from the ridiculously large horizontal DA of the R. FODO to the small vertical DA of the FODO. One is tempted to conclude from this table that the R. FODO would be the best choice.

TABLE 1. The dynamic aperture of the three ideal lattice variants. The DA is shown in units of $\mathrm{cm}$ along the horizontal, diagonal, and vertical directions in the $x-y$ plane.

\begin{tabular}{cccc}
\hline Lattice / Direction & $\mathbf{0}^{\circ}$ & $\mathbf{4 5}^{\circ}$ & $\mathbf{9 0}^{\circ}$ \\
\hline FODO & 17.5 & 10.0 & 6.5 \\
R. FODO & $\approx 150$ & 19.0 & 15.5 \\
PD & 30.5 & 13.5 & 15.0 \\
\hline
\end{tabular}

However, we will see in the next section that this is misleading. The first clue that some "accidental cancellation" is causing this behavior is obtained by looking at the dynamic aperture of the ideal lattices with the horizontal sextupoles turned off. The results are shown in Table 2. The table is structured in the same way as Table 1. One expects that in the absence of the nonlinearities due to the sextupoles, the DA would increase or at least stay unchanged. This is indeed the case in the horizontal direction, but in the case of the vertical direction of the PD lattice the DA decreases by 33\%, which implies cross-talking of the horizontal and vertical planes due to the horizontal sextupoles. Also, a small reduction in the diagonal DA of the R. FODO can be observed. This in turn implies that some accidental cancellations are going on in the ideal lattices, which might not be robust against systematic and/or random multipole errors. This is the case studied in the next section.

\section{Lattices with Errors}

A set of realistic multipole errors was included in the simulation of all three ideal lattices (with the horizontal sextupoles now turned back on), taken from the Fermilab's Main Injector Technical Design Handbook (see Table 3) [6]. Only normal systematic dipole and quadrupole 
TABLE 2. The dynamic aperture of the three ideal lattice variants with horizontal sextupoles turned off. The DA is shown in units of $\mathrm{cm}$ along the horizontal, diagonal, and vertical directions in the $x-y$ plane. To be compared with Table 1 .

\begin{tabular}{cccc}
\hline Lattice / Direction & $\mathbf{0}^{\circ}$ & $\mathbf{4 5}^{\circ}$ & $\mathbf{9 0}^{\circ}$ \\
\hline FODO & $\approx 110$ & 10.5 & 8.0 \\
R. FODO & $\approx 182$ & 18.5 & 15.5 \\
PD & $\approx 131$ & 14.0 & 10.0 \\
\hline
\end{tabular}

body errors have been used, the dipole errors up to 14poles, and quadrupole errors up to 20-poles. We note that the conclusions of these studies are not very sensitive to the magnitude of the errors, so one can think of the conclusions as being valid for any generic set of multipole magnet errors.

TABLE 3. Set of multipole magnet errors used in the tracking, taken from the Fermilab's Main Injector Technical Design Handbook.

\begin{tabular}{ccc}
\hline Multipole order & \multicolumn{2}{c}{ Error $<\mathbf{b}_{\mathbf{n}}>$} \\
\cline { 2 - 3 } & Dipole & Quadrupole \\
\hline 4 & 0.06 & - \\
6 & -0.4 & 0.5 \\
8 & 0.04 & 5.85 \\
10 & 0.33 & -0.1 \\
12 & -0.01 & -1.82 \\
14 & -0.03 & 0.21 \\
16 & & 1.41 \\
18 & & -0.03 \\
20 & & -0.8 \\
\hline
\end{tabular}

Under these conditions the DAs of all three lattices change dramatically compared to the ideal lattices. Now essentially all lattices have the same, reduced, DA, as presented in Table 4, with the notable difference of the horizontal DA of the PD, which might be significantly above the noise level. Clearly, the errors now dominate the DA, and any cancellation or point solution that was present in the ideal lattices vanished. Since errors in practice are unavoidable, it follows that realistically there is no clear best lattice in terms of dynamic aperture.

TABLE 4. The dynamic aperture of the three lattice variants with multipole errors turned on. The DA is shown in units of $\mathrm{cm}$ along the horizontal, diagonal, and vertical directions in the $x-y$ plane. To be compared with Table 1.

\begin{tabular}{cccc}
\hline Lattice / Direction & $\mathbf{0}^{\circ}$ & $\mathbf{4 5}^{\circ}$ & $\mathbf{9 0}^{\circ}$ \\
\hline FODO & 3.5 & 1.5 & 1.5 \\
R. FODO & 3.5 & 1.5 & 1.5 \\
PD & 6.0 & 2.0 & 1.5 \\
\hline
\end{tabular}

\section{ANALYSIS OF THE RESULTS}

Since the tracking was performed with the one-turn map, the order-by-order amplitude dependent tune shifts and resonance strengths can be analytically extracted using Differential Algebraic normal form techniques [7]. Their relative magnitude sheds light on the reasons behind the widely varying dynamic apertures. We exemplify the process through a few examples.

For example, why is the horizontal DA of the ideal rearranged FODO so large compared to the other two? Looking at first and second order amplitude dependent tune shifts we obtained Table 5. The reason is clear now; the FODO has very large higher order amplitude dependent tune shifts.

TABLE 5. Comparison of first and second order amplitude dependent coefficients of two ideal lattices.

\begin{tabular}{ccc}
\hline Tune shift coef. & R. FODO & FODO \\
\hline First order & -0.2 & -8 \\
Second order & -2 & 11828 \\
\hline
\end{tabular}

Or one might wonder why does the vertical DA stay the same for the rearranged FODO and is decreasing for PD, when the horizontal sextupoles are turned off? Again, a quick look at tune shifts solves the puzzle (see Table 6).

TABLE 6. Comparison of first and second order amplitude dependent coefficients of two ideal lattices with horizontal sextupoles turned on and off, respectively.

\begin{tabular}{ccccc}
\hline Tune shift c. & \multicolumn{2}{c}{ R. FODO } & \multicolumn{2}{c}{ PD } \\
\hline Horizontal & HS ON & HS OFF & HS ON & HS OFF \\
\hline First order & -2 & -2 & -7 & -1 \\
Second order & 6548 & 6385 & -28516 & -8144 \\
\hline Vertical & & & & \\
\hline First order & 0.1 & -0.1 & 2 & -6 \\
Second order & 154 & 188 & -19 & 654 \\
\hline
\end{tabular}

Finally, the question arises why are the properties of the three lattices essentially the same when magnet errors are included? For example, the largest tune shift coefficients are roughly equal, as seen in Table 7. Likewise, the dominating resonance driving terms, included in Table 8 are also the same and of the same order of magnitude. However, notice that at third order the $(1,0)$ resonance is not excited in the case of PD, which is the reason why PD has a larger horizontal dynamic aperture.

Finally, we checked also higher order tune shifts and resonance strengths, and the same qualitative aspects persist to very high orders. 
TABLE 7. Comparison of the largest first and second order amplitude dependent coefficients of the three lattices with multipole errors on.

\begin{tabular}{cccc}
\hline Tune shift coef. & FODO & R. FODO & PD \\
\hline First order & 144 & 94 & 150 \\
Second order & 4161514 & 3317155 & 2346097 \\
\hline
\end{tabular}

TABLE 8. Comparison of the dominating resonance driving terms of the three lattices with multipole errors on.

\begin{tabular}{cccc}
\hline D. t. c. & FODO & R. FODO & PD \\
\hline 3rd order & of $(1,0):: 28$ & of $(1,0):: 22$ & of $(3,0):: 12$ \\
4th order & $(2,-2):: 18217$ & 14605 & 33198 \\
5th order & $(1,0):: 1160894$ & 1174164 & 946206 \\
\hline
\end{tabular}

\section{CONCLUSIONS}

Three variants of a Proton Driver lattice have been investigated for dynamic aperture with emphasis on robustness studies with respect to multipole errors. Ideal lattices showed a wide array of properties, mainly due to the positioning of the sextupoles. However, the "accidental" cancellations of the ideal lattices in general did not survive under the presence of generic magnet errors, and the lattices with errors showed essentially the same behavior (with PD performing a tad better for the set of errors employed in this study).

Although no systematic study has been done, it seems that the choice of the tune alters the results quantitatively, but qualitatively the same conclusions can be drawn.

\section{REFERENCES}

1. Makino, K., and Berz, M., Nuclear Instruments and Methods, A427, 338-343 (1999).

2. Berz, M., COSY INFINITY Version 8 reference manual, Tech. Rep. MSUCL-1088, National

Superconducting Cyclotron Laboratory, Michigan State University, East Lansing, MI 48824 (1997), see also http://www.beamtheory.nscl.msu.edu/cosy.

3. M. Berz et al., The COSY INFINITY web page, http://www.beamtheory.nscl.msu.edu/cosy.

4. Erdelyi, B., and Berz, M., Phys. Rev. Lett., 87, 114302 (2001).

5. Erdelyi, B., Ph.D. thesis, Michigan State University (2001).

6. Fermilab Main Injector Department, The Fermilab Main Injector Technical Design Handbook, http://wwwfmiinternal.fnal.gov/MI_Technical_Design/index.html (1994).

7. Berz, M., "Differential Algebraic Formulation of Normal Form Theory," in M. Berz, S. Martin and K. Ziegler (Eds.), Proc. Nonlinear Effects in Accelerators, IOP Publishing, London, 1992, p. 77. 